\title{
USOS E LIMITAÇÕES DO MÉTODO LADDERING
}

\section{LADDERING METHOD: USES AND LIMITATIONS}

TÂNIA MODESTO VELUDO-DE-OLIVEIRA Mestre em Administração pela Faculdade de Economia, Administração e Contabilidade da Universidade de São Paulo (FEA/USP). Professora da Fundação Escola de Comércio Álvares Penteado (Unifecap).

Rua Joaquim Antunes, 553, apto. 102, São Paulo - SP - CEP 05415-011

E-mail: tveludo@usp.br

A NA AKEM I IKEDA Professora associada e coordenadora da área de Marketing da Faculdade de Economia, Administração e Contabilidade da Universidade de São Paulo (FEA/USP). Vice-coordenadora do MBA Marketing da FIA/FEA/USP. Av. Prof. Luciano Gualberto, 908, sala e-104, São Paulo - SP - CEP 05508-900 E-mail: anaikeda@usp.br 


\section{RESUMO}

Este artigo tem por objetivo apresentar o método laddering e discutir as possibilidades de sua aplicação em pesquisas acadêmicas e de mercado, para contribuir com a disseminação de técnicas metodológicas que se apresentem como novas alternativas à pesquisa no Brasil. Foi realizado um levantamento bibliográfico a partir do qual descreveu-se o método, seu surgimento e suas etapas. Além disso, discutiram-se algumas de suas vertentes (hard laddering e LADDERMAP) e suas principais limitações. Conclui-se que o laddering é um método de grande valia no estudo do comportamento humano, servindo, sobretudo, à avaliação de valores dos indivíduos no contexto da teoria de cadeias meios-fim. No emprego do laddering, recomenda-se a observação de alguns pontos, como a qualificação do entrevistador e do pesquisador, a homogeneização do grupo de respondentes, o uso do software LADDERMAP para facilitar a operacionalização do método e a consideração de critérios na escolha do hard ou soft laddering.

\section{PA L A V A S-CHA V E}

Laddering; Metodologia; Cadeias meios-fim; Valor; Comportamento.

\section{A B STRACT}

This article aims at showing the laddering method, considering the possibilities of its utilization in both academic and market researches. The idea is to contribute with the dissemination of methodological techniques that are new alternatives to the researches in Brazil. The literature review allowed to describe the method, its emergence and stages. Moreover, there is a discussion about hard laddering and LADDERMAP and their main limitations. The conclusion is that laddering method is of good value to human behavior studies, and above all to individual evaluation in the context of means-end chain theory. Authors emphasize that laddering demands a good interviewer and researcher, a homogeneous group to answer the questions and the use of LADDERMAP software to ease its application. Finally, some attention has to be given to the criteria of choosing hard or soft laddering.

\section{KEYWORDS}

Laddering; Methodology; Means-end chain; Value; Behavior. 


\section{I NTRODUÇÃ O}

O laddering surgiu como uma ferramenta para se tentar compreender o significado de certos comportamentos. Esse método vem sendo utilizado na área de administração e marketing para investigar opiniões, atitudes e crenças de indivíduos, sendo bastante recomendado em pesquisas que abordam o valor para o cliente segundo os moldes da teoria de cadeias meios-fim (REYNOLDS; GUTMAN, I988; VALETTE-FLORENCE; RAPACCHI, I99I; GENGLER; REYNOLDS, I995; REYNOLDS; WHITLARK, I995; LASTOVICKA, I995; WOODRUFF; GARDIAL, I996; BOTSCHEN; THELEN; PIETERS, I999; GENGLER; MULVEY; OGLETHORPE, I999; VRIENS; HOFSTEDE, 2000; WANSINK, 2000; DIBLEY; BAKER, 200I; CHI-FENG, 2002). O modelo de meios-fim une seqüencialmente, em uma hierarquia de valor, os atributos de um produto $(\mathrm{A})$ às conseqüências de uso do produto $(\mathrm{C})$ e aos valores pessoais dos indivíduos $(\mathrm{V})$, formando uma cadeia (ladder, seqüência A-C-V ou cadeia meios-fim).

Apesar de sua repercussão internacional, parece que no Brasil esse método ainda está em processo de disseminação, pois apenas recentemente alguns trabalhos começaram a ser desenvolvidos segundo seus preceitos, como o de Leão e Mello (2001, 2002), Veludo-de-Oliveira (2003) e Ignácio (2003). Assim, este artigo tem por objetivo apresentar o método laddering e discutir as possibilidades de sua aplicação em pesquisas acadêmicas e de mercado, para contribuir com a disseminação de técnicas metodológicas que se apresentem como novas alternativas à pesquisa no Brasil. Para isso, realizou-se um levantamento bibliográfico que subsidiou a descrição do laddering e de suas etapas, bem como a apresentação de algumas variantes do método e suas limitações.

\section{O MÉTODO LADDERING: SURGIMENTO E DEFINIÇÕES}

Dibley e Baker (2001:78) remontam, sucintamente, o surgimento do método laddering. Eles contam que o método teve origem no trabalho de Kelly (I955) que, como pesquisadora do campo da psicoterapia, elaborou uma teoria geral (Personal Construct Theory) baseada em 25 anos de clínica, para interpretar e antecipar as experiências dos indivíduos. Esse trabalho contribuiu para o avanço da interpretação cognitiva e para a crença de que um conjunto de processos internos - pensamentos, imagens, construtos - são responsáveis pela conduta das pessoas. E foi Hinkle (1965), discutido por Bannister e Mair (I968), que desenvolveu a técnica laddering como um meio de acessar os sistemas de 
significado pessoais de indivíduos, sendo essa uma técnica essencialmente qualitativa, que exige que os respondentes façam abstrações. No domínio de marketing e em particular no de comportamento do cliente, a interpretação de Gutman (I982) para compreender a cognição é conhecida como teoria de cadeias meios-fim e foi o seu trabalho, desenvolvido em conjunto com Reynolds (REYNOLDS; GUTMAN, I988), que disseminou a idéia da aplicação do laddering na área de marketing. Reynolds e Gutman (I988:I2) definem a técnica laddering:

Laddering se refere a uma técnica de entrevista em profundidade, individual, usada para compreender como os clientes traduzem o atributo de produtos em associações com significado a respeito de si mesmos, seguindo a teoria de cadeias meios-fins.

Além de orientar a coleta de dados primários, o laddering também envolve procedimentos para análise e interpretação dos dados. Gengler e Reynolds (I995: 2I) resumem os passos do método da seguinte maneira:

a) Quebra dos dados, conversão dos dados em frases separadas. Essas frases são os elementos básicos nos quais as análises subseqüentes estão baseadas. Isso envolve revisão em anotações e em fitas de discussão, [para identificar] os elementos que melhor representam os conceitos expressos por cada sujeito individualmente;

b) Análise de conteúdo dos elementos selecionados no passo a);

c) Realização de associações entre códigos de conteúdo, resultando na avaliação quantitativa de todos os relacionamentos pareados, chamadas implicações;

d) Construção de um diagrama para representar significativamente as principais implicações, chamado mapa hierárquico de valor (HVM).

\section{O MÉTODO LADDERING E SUAS ETAPAS}

O laddering se desdobra em duas etapas principais: (i) coleta de dados; e (ii) análise e interpretação dos dados. Cada uma delas é enfatizada a seguir. 


\section{1 COLETA DE DADOS}

O laddering tece uma série de orientações para a coleta de dados primários, destacando alguns procedimentos a serem seguidos durante a entrevista. Wansink (2000) faz um paralelo entre uma entrevista laddering e o trabalho de um psicólogo, pois a técnica auxilia a descoberta de insights, da mesma forma que esse profissional o faz com aspectos que não são aparentes nem ao paciente.

No laddering, inicialmente, pergunta-se ao entrevistado que tipos de características seriam úteis para descrever ou distinguir diferentes marcas ou produtos de uma dada categoria de interesse. O objetivo desse primeiro passo é fazer com que o respondente mencione os principais atributos do produto, o que, segundo Reynolds e Gutman (I988), é obtido de três formas. A primeira refere-se a uma técnica chamada "escolha de três" (triadic sorting), em que são apresentadas três marcas distintas de um produto e solicita-se ao entrevistado indicar diferenças e similaridades que duas marcas possuem em relação à terceira. A outra técnica, denominada "diferenças de preferência de consumo", pede ao entrevistado que indique por que uma marca é preferível em relação às outras. A última técnica recomendada é chamada "diferenças de ocasião". Nela, o cliente é inserido em um contexto de consumo, em que descreve as características relativas a esse consumo.

Deve-se ressaltar que há possibilidade de que as respostas para esse questionamento inicial, em vez de se relacionarem a atributos, se refiram a conseqüências ou a valores pessoais. Entretanto, Woodruff e Gardial (I996) argumentam que a experiência tem mostrado que provavelmente as respostas iniciais farão referência a atributos e que as demais dimensões da hierarquia de valor deverão ser construídas a partir da indicação dessas características. Corrobora essa afirmação Wansink (2000), colocando que as respostas, nessa fase da investigação, tipicamente descrevem as características dos produtos, como cor, gosto, preço, tamanho, entre outras.

A partir da resposta inicial dos entrevistados, que se refere a atributos, começa a ser construída a hierarquia de valor, quando o pesquisador discute as razões das preferências apontadas pelo respondente e, com isso, consegue levar as respostas a um nível de abstração correspondente às conseqüências e valores pessoais.

$\mathrm{Na}$ entrevista laddering, os respondentes são encorajados, por meio de perguntas repetidas e interativas, a se aprofundar na discussão sobre os atributos, indicando, paulatinamente, conseqüências e valores pessoais. O pesquisador conduz o entrevistado à abstração, por meio de questionamentos sobre por que tal atributo (ou conseqüência) é importante. Nesse sentido, questões do 
tipo "Por que isso é importante?", "O que isso significa para você?” e "Qual é o significado de o produto possuir (ou não) esse atributo?” são feitas de maneira repetitiva aos entrevistados com o objetivo de fazê-los expressar as conseqüências que são derivadas dos atributos e os valores pessoais que se originam das conseqüências. Wansink (2000) lembra que as questões são continuamente feitas até um valor pessoal ser revelado. Nesse sentido, a entrevista laddering é bastante personalizada, pois depende essencialmente das respostas dos entrevistados para seguir adiante, tendo como ponto de referência a pergunta "Por que é importante?".

Woodruff e Gardial (I996) advertem que as perguntas repetitivas podem fazer com que as questões sejam um tanto quanto óbvias ao respondente, além de tornar a investigação cansativa. Por essa razão, é importante informar o entrevistado das peculiaridades da técnica, deixando claro que os procedimentos adotados são parte de uma metodologia específica.

Botschen, Thelen e Pieters (I999) comentam que uma crítica comum à abordagem de cadeias meios-fim é que, ao se perguntar "Por quê?", podem ocorrer níveis de abstração "artificiais", pois os entrevistados podem responder de maneira "racional", tentando encontrar argumentos para justificar seu comportamento.

Reynolds e Gutman (I988) ainda apontam outros problemas advindos desse tipo de entrevista. Um deles se relaciona à possibilidade de o entrevistado não saber responder a uma questão, por nunca haver pensado sobre ela ou por não conseguir refletir sobre as razões de sua importância. Outro problema pode ser causado pelo fato de as questões se tornarem demasiadamente pessoais e, com isso, intimidarem a expressão verdadeira do respondente. Um sinal de que isso está acontecendo é quando o respondente não evolui o nível de sua resposta, insistindo em mantê-la num patamar igual ou mesmo inferior ao que estava. Outros indícios desse problema aparecem quando o respondente diz que não sabe responder à questão, fica em silêncio ou usa argumentos que, apesar de girarem em torno da pergunta, não lhe responde satisfatoriamente.

Em relação aos problemas identificados, Reynolds e Gutman (I988) propõem algumas técnicas para solucioná-los ou, ao menos, minimizá-los. Entre elas, destacam-se:

- evocar um contexto situacional: essa técnica consiste em fazer a pergunta considerando uma situação específica, pois os entrevistados têm mais facilidade de responder a uma questão quando imaginam um contexto circunstancial. É possível obter melhores resultados com o laddering quando os respondentes fazem associações a partir de uma ocasião real na qual eles podem usar o produto; 
- postular a falta de um objeto: essa técnica é empregada para desbloquear o respondente quando ele não consegue se mover além de certo nível da hierarquia. Consiste em encorajá-lo a considerar o que ele faria na falta do objeto, supondo que o respondente usará argumentos de "substituição" ao se imaginar sem uma característica do produto ou sem o produto ou sem uma conseqüência de uso;

- laddering negativo: essa técnica é interessante quando o respondente não consegue explicar as razões por que age de determinada maneira. Em vez de perguntar a ele por que faz ou pensa de determinada forma, pergunta-se por que ele não faria ou não pensaria desse jeito;

- contraste com a regressão da idade: essa técnica consiste em fazer o respondente voltar no tempo e relembrar seus hábitos passados, para compará-los com os atuais;

- abordagem de terceira pessoa: essa técnica é utilizada com o intuito de deixar o respondente mais à vontade para comentar suas opiniões e consiste em fazê-lo imaginar como outra pessoa agiria ou se sentiria em dada situação. $\mathrm{Na}$ verdade, dessa forma ele expressa a própria maneira de agir e sentir;

- técnicas de redirecionamento (silêncio e checagem da comunicação): essas técnicas consistem em direcionar novamente a resposta ao entrevistado, fazendo silêncio para que ele complete seu raciocínio sem maiores interferências ou fazendo uma verificação de comunicação, em que se repete a resposta do entrevistado e pede-se para que ele esclareça o que quis dizer com ela.

Reynolds e Gutman (1988) ainda evidenciam duas outras técnicas no caso de o respondente não se sentir à vontade com as questões por estarem se tornando muito pessoais. Uma das técnicas se refere à revelação de um fato pessoal pelo pesquisador, em uma demonstração de cumplicidade para com o entrevistado. Contudo, eles avisam que essa pode ser uma opção perigosa e lembram que esse fato pessoal pode ser, inclusive, "fabricado". Neste artigo, chama-se a atenção para o aspecto ético que permeia o uso da técnica citada. A outra técnica, comumente empregada, é utilizada para que o respondente não se sinta pressionado e consiste em deixar momentaneamente o assunto de lado para retomá-lo em um momento posterior da entrevista.

Enfim, Wansink (2000) condensa os principais pontos que devem ser evitados e priorizados em uma entrevista laddering, destacando o "faça e não faça" (Quadro I). 


\section{QUADRO I}

PONTOS QUE DEVEM SER EVITADOS OU PRIORIZADOS EM UMA ENTREVISTA LADDERING

\section{Faça}

Não faça

- Perguntas que possam revelar razões pes- - Não tenha pressa! soais.

- Não faça perguntas que possam ser

- Perguntas que permitam que a pessoa respondidas com uma única palavra. pense e responda com uma frase, e não - Não force o entrevistado a responder apenas com um "sim" ou "não". à questão de certo modo.

- Várias vezes a pergunta "Por quê?”.

- Não espere desvendar um valor com

- Perguntas sobre as razões das pessoas para suas respostas. apenas três questões.

- Permita que o questionamento flua, até se - Não assuma que uma pessoa quis as questões não forem diretamente relativas à marca. dizer algo que ela não disse.

- Não force a questão. Algumas con-

- Perguntas que dão liberdade ao entrevistado para responder à questão como ele acha mais adequado. seqüências podem não levar aonde você quer chegar. Mude os tópicos e comece de novo.

- Não desanime!

- Perceba gestos, expressões faciais e como as pessoas respondem à questão e ouça o tom de voz delas.

Fonte: Wansink (2000:35).

Deve-se, finalmente, destacar a importância da figura do entrevistador nessa fase da investigação, que requer "entrevistadores com habilidades qualitativas" (VRIENS; HOFSTEDE, 2000:6). Wansink (2000) afirma que algumas vezes as questões certas não aparecem facilmente e que o respondente pode se sentir nervoso ou desconfortável com a linha das perguntas, o que demanda habilidade do pesquisador ao conduzi-las. Segundo ele (p. 32), "a chave para se conduzir uma entrevista laddering com sucesso é a prática” e para isso é preciso tempo e paciência.

\subsection{ANÁLISE E INTERPRETAÇÃO DOS DADOS}

A análise dos dados de uma investigação conduzida por meio do laddering conta com quatro etapas consecutivas: (a) análise de conteúdo; (b) desenvolvimento da matriz de implicação; (c) construção do mapa hierárquico de valor; e (d) determinação das orientações de percepção dominantes. Cada uma das etapas é discutida a seguir. 


\section{a) Análise de conteúdo}

O material obtido, em geral, a partir de transcrições de fitas de gravação com as entrevistas deve ser submetido à análise de conteúdo - "técnica de observação usada para analisar unidades de material escrito por meio de regras cuidadosamente aplicadas" (AAKER; KUMAR; DAY, 2001:223).

O laddering não recomenda o uso de técnicas específicas de análise de conteúdo, que se inicia com uma leitura cuidadosa dos dados para identificação de passagens no texto que reflitam aspectos importantes e centrais do que foi expresso. Esse processo consiste na leitura de cada parte do texto, seguida pela identificação de uma oração que a represente. Frases com um mesmo sentido são alocadas sob uma mesma oração. Após o desmembramento, devem-se classificar os elementos em atributos, conseqüências e valores pessoais.

O resultado dessa etapa é um conjunto de elementos (orações ou de palavras-chave) relacionadas em uma seqüência $\mathrm{A}-\mathrm{C}-\mathrm{V}$, que expressam, de forma resumida, o "raciocínio" dos indivíduos ao associar atributos, conseqüências e valores pessoais. Ao final, têm-se várias seqüências $A-C-V$, resultantes das entrevistas com os respondentes. Vale ressaltar, no entanto, que nem sempre é possível construir cadeias meios-fim a partir de todas as entrevistas. Reynolds e Gutman (I988) afirmam que é normal que até um quarto das entrevistas não se constituam em ladders. Para $75 \%$ dos entrevistados, é possível identificar de duas a três seqüências A-C-V (REYNOLDS; GUTMAN, I988; DIBLEY; BAKER, 200I).

O próximo passo é a codificação desses elementos, a partir da atribuição de números que, em uma ordem crescente, se referem a atributos, conseqüências e valores pessoais. Assim, o elemento número i, provavelmente, se refere a um atributo que representa o nível mais baixo da cadeia.

Gengler, Mulvey e Oglethorpe (I999) alertam que o objetivo da análise de conteúdo não é retratar os ladders de cada pessoa e sim desenvolver uma representação agregada e fiel à estrutura de pensamento das pessoas entrevistadas. Mais uma vez, a figura do pesquisador merece ser realçada. Nessa etapa, o trabalho é intensivo e necessita de cuidado e habilidade para ser realizado, pois os resultados da análise de conteúdo servem de base para as análises subseqüentes. "A análise de conteúdo é uma tarefa interativa, em que o analista pode re-codificar os dados várias vezes, combinar categorias, dividi-las, eliminá-las ou criar novas, até sentir que alcançou uma solução ótima" (GENGLER; REYNOLDS, I995:22). O resultado final dessa etapa é condensado em um quadro-resumo, com os principais elementos oriundos das entrevistas, classificados e codificados dentro da hierarquia de valor. O Quadro 2 exemplifica a identificação dos elementos de uma cadeia meios-fim para consumidores de vinho carbonado. 
QUADRO 2

RESUMO DE CÓDIGOS DE CONTEÚDO (REPRESENTATIVO)

\begin{tabular}{|c|c|c|c|c|}
\hline Cód. & Atributos & Cód. & Conseqüências & Cód. Valores pessoais \\
\hline OI & Carbonação & 08 & Qualidade & 20 Realização \\
\hline 02 & Seco & 09 & Satisfaz & 2I Vida familiar \\
\hline 03 & Caro & IO & Refrescante & 22 Pertencimento \\
\hline 04 & Rótulo & II & Consome menos & 23 Auto-estima \\
\hline \multirow[t]{2}{*}{05} & Formato da & $\mathrm{I} 2$ & Mata a sede & \\
\hline & garrafa & I3 & Mais feminina & \\
\hline 06 & Menos álcool & I4 & Evita os efeitos negativos do álcool & \\
\hline \multirow[t]{5}{*}{07} & Tamanho menor & I5 & Evita o desperdício & \\
\hline & da garrafa & 16 & Recompensa & \\
\hline & & I7 & Imagem sofisticada & \\
\hline & & I8 & Impressiona os outros & \\
\hline & & I9 & Capaz de socializar & \\
\hline
\end{tabular}

\section{b) Desenvolvimento da matriz de implicação}

A construção da matriz de implicação é o segundo passo na análise do método laddering. Todos os elementos selecionados na primeira fase são alocados, por meio de seus códigos, nas linhas e nas colunas de uma tabela numérica, formando uma matriz. Nessa fase, são analisadas as relações entre os elementos, por meio da verificação de quantas vezes dado elemento leva a outro. Para a contagem das relações existentes entre os elementos, é necessário analisar os ladders das entrevistas de cada respondente. São também considerados os tipos de relação entre os elementos, pois eles podem relacionar-se de forma direta, quando são adjacentes, ou de forma indireta, quando há outros elementos entre eles. A quantidade de relações entre os elementos é apresentada na matriz na forma fracional, em que as relações diretas $(\mathrm{XX})$ aparecem à esquerda do ponto e as indiretas (YY) estão à direita do ponto final.

Leão e Mello (200I) alertam para algumas dúvidas que podem surgir na contagem do número de relações para a construção da matriz. A dúvida levantada é se as relações que foram mencionadas mais de uma vez pelo mesmo entrevistado devem ser consideradas apenas uma única vez ou se a contagem deve levar em conta cada uma dessas menções. Os autores (200I:Io) citam um exemplo para esclarecer a questão.

Para exemplificar este impasse, vamos tomar como referência dois ladders quaisquer (hipotéticos) de um mesmo entrevistado: 6-I7 - I9 ou 6-I7-28. A dúvida é se a relação 6 - I7 deve ser contada duas vezes (uma vez referente a cada ladder) ou apenas uma (por ser de um mesmo entrevistado). 
Gengler (I995) explica que o ideal é contar a relação apenas uma vez para não enviesar os resultados. O resultado dessa fase é a matriz de implicação (Tabela I), que expressa um exemplo referente aos vinhos carbonados. Assim, analisando a primeira linha da Tabela I, depreende-se que apenas um entrevistado associou o elemento I (carbonação) diretamente ao elemento 8 (qualidade) e ninguém associou esses elementos indiretamente, pois a célula formada pela junção do elemento I (carbonação) e do elemento 8 (qualidade) é I.oo. Analogamente, o elemento I (carbonação) foi associado ao elemento io (refrescante) dez vezes diretamente e nenhuma vez indiretamente, formando a célula io.০o.

\section{TABELA I}

MATRIZ DE IMPLICAÇÃO (REPRESENTATIVA)*

\begin{tabular}{|c|c|c|c|c|c|c|c|c|c|c|c|c|c|c|c|c|}
\hline Cód. & o8 & 09 & IO & II & 12 & I3 & I4 & 15 & 16 & I7 & I8 & 19 & 20 & $2 \mathrm{I}$ & 22 & 23 \\
\hline OI & I.०O & & 10.00 & & 4.06 & & & .OI &. $\mathrm{I} 4$ & & .04 & & .06 & & & .04 \\
\hline 02 & 3.00 & & 4.00 & & .04 & & & & .04 & .03 & .04 & $.0 \mathrm{I}$ & & & .07 & \\
\hline 03 & I2.00 & & & & & & & & 2.04 & I.OI & 1.09 & & 1.06 & & .05 & .05 \\
\hline 04 & 2.00 & & & & & 2.02 & & & & 2.04 & .02 & & .OI & & .02 & .03 \\
\hline 05 & I.०० & & I.०० & & & 2.02 & & & & I.03 & & & & & .02 & .03 \\
\hline 06 & & & I.०० & & I.०० & & 5.00 & & $.0 \mathrm{I}$ & & $.0 \mathrm{I}$ & I.OI & & .04 & .OI & \\
\hline 07 & & & & I.०O & & & .OI & 3.00 & & & & .01 & & .02 & .OI & \\
\hline 08 & & & & & & 3.00 & & I.OO & 4.00 & 4.03 & 4.04 & .OI & 3.02 & & .09 & .04 \\
\hline 09 & & & & 4.00 & & & .04 & & & & & & I.03 & & .03 & .02 \\
\hline I0 & & & & & 10.00 & I.00 & & & 5.10 & .OI & .06 & & .04 & & .05 & .02 \\
\hline II & & & & & & & 5.00 & & & & & .04 & & .02 & .03 & \\
\hline I2 & & & & & & & & & I4.00 & & .08 & & .06 & & .04 & .04 \\
\hline I3 & & & & & & & & & & 7.00 & .02 & & & & I.O3 & .04 \\
\hline I4 & & & & & & & & & & & I.00 & 5.00 & & 4.01 & .04 & \\
\hline I5 & & & & & & & & & & & & & & 2.00 & & \\
\hline I6 & & & & & & & & & & & II.०० & & 8.00 & & .06 & 1.05 \\
\hline I7 & & & & & & & & & & & 4.00 & I.०० & I.०० & & 4.02 & 5.03 \\
\hline I8 & & & & & & & & & & & & 1.00 & I.०० & & 10.00 & 9.00 \\
\hline 19 & & & & & & & & & & & & & & 3.00 & 5.00 & \\
\hline 20 & & & & & & & & & & & & & & & & \\
\hline $2 \mathrm{I}$ & & & & & & & & & & & & & & & & \\
\hline 22 & & & & & & & & & & & & & & & & \\
\hline 23 & & & & & & & & & & & & & & & & \\
\hline
\end{tabular}

Fonte: Reynolds e Gutman (I988:2I).

* Na tabela, as relações diretas aparecem à esquerda do ponto e são representadas por "XX" e as indiretas estão à direita e são representadas por "YY". As relações entre os elementos são, portanto, apresentadas na forma "XX.YY". 


\section{c) Construção do mapa hierárquico de valor (HVM)}

A idéia dessa etapa é mapear as relações existentes entre os elementos, sejam elas relações diretas ou indiretas. Todavia, nem todas as relações entre atributos, conseqüências e valores pessoais são consideradas, e sim as mais importantes, que, por sua vez, são filtradas com o estabelecimento de um ponto de corte, que se refere a um número mínimo de vezes em que as relações diretas e indiretas aparecem na matriz de implicação. As relações que não atingem o ponto de corte são descartadas. O ponto de corte ideal é o que leva o mapa a cobrir, pelo menos, dois terços das relações apresentadas na matriz de implicação (REYNOLDS; GUTMAN, Ig88).

A construção do mapa implica a verificação dos tipos de relações existentes entre os elementos, que podem ser: elementos adjacentes (A-D), elementos não adjacentes (N-D), elementos adjacentes com muitas relações indiretas (AI), elementos não adjacentes com muitas relações indiretas (N-I) e, por fim, elementos não adjacentes com baixo número de relações (N-O) (REYNOLDS; GUTMAN, I988).

O processo de construção do mapa consiste na estruturação de cadeias meios-fim, partindo da primeira linha da matriz de implicação e indo até a última. Tendo em vista a primeira linha da matriz, deve-se procurar a primeira coluna com um número de relações diretas ou indiretas superiores ao ponto de corte estabelecido. O encontro da célula que combina a primeira linha com tal coluna forma o início de uma seqüência $\mathrm{A}-\mathrm{C}-\mathrm{V}$. Para dar continuidade à seqüência, movimenta-se para baixo, indo até a linha que contém o número dessa coluna. No exemplo dos vinhos carbonados, o ponto de corte estabelecido foi 4, a coluna Io (refrescante) é a primeira a estabelecer relações com a linha I (carbonação). Essas relações são diretas (o número Io está antes do ponto final) e superiores ao ponto de corte, pois io (I0.00) é maior que 4. Então, deve-se partir até a linha io (refrescante) e verificar nela qual é a coluna que primeiro mostra relações superiores ao ponto de corte, formando, assim, mais um caminho da seqüência A$\mathrm{C}-\mathrm{V}$. No exemplo dos vinhos carbonados, essas relações são estabelecidas com a coluna I2 (mata a sede), que também apresenta relações diretas com o elemento Io (refrescante). Isso forma a seqüência "carbonação (I)-refrescante (IO)-mata a sede (i2)". Esse processo deve continuar até que a última linha seja alcançada.

Reynolds e Gutman (I988) preceituam que o objetivo dessa etapa é inter-relacionar as cadeias significativas, de modo a facilitar sua interpretação, de preferência, evitando o cruzamento de linhas. Esse procedimento indica todas as relações importantes entre atributos, conseqüências e valores pessoais. Após a construção do mapa, é feito um resumo com as relações diretas (XX) e indiretas (YY) de cada elemento (XX.YY). O resumo deve apresentar a quantidade de relações que "partiram" de cada elemento ou que "vieram" até ele. A relevância desse resumo está 
em identificar facilmente quais são os elementos mais importantes em cada nível. Essa etapa da análise deve originar o mapa hierárquico de valor (HVM), cuja estrutura é representada pela Figura i e numa tabela-resumo, indicando o número total de relações diretas e indiretas entre os elementos, como apresenta a Tabela 2.

O desenho i e a Tabela 2 estão baseados no exemplo dos vinhos carbonados. Na Tabela 2, o número total de relações diretas que partem do elemento I (carbonação) é I5, somando-se "I" da coluna 8, "IO" da coluna io e "4" da coluna I2. Seguindo a mesma lógica, o número de relações indiretas que partem do elemento I (carbonação) é 35, pois soma-se "6" da coluna I2, "I" da coluna I5, "I4" da coluna I6, "4" da coluna I8, "6" da coluna 20 e "4" da coluna 23. Por outro lado, nenhuma relação chega ao elemento I, pois dele apenas partem interações com outros elementos.

\section{DESENHO I}

\section{ESTRUTURA DO MAPA HIERARQUICO DE VALOR (REPRESENTATIVA)}



Fonte: Reynolds e Gutman (I988:I9). 


\section{TABELA 2}

RESUMO DE RELAÇOES DIRETAS (XX) E INDIRETAS (YY)

DE CADA ELEMENTO (XX.YY) (REPRESENTATIVA)

\begin{tabular}{|c|c|c|}
\hline Código & De & Para \\
\hline OI & I5.35 & 0.00 \\
\hline $\mathrm{O} 2$ & 7.23 & 0.00 \\
\hline 03 & I7.30 & 0.00 \\
\hline 04 & 6.14 & 0.00 \\
\hline 05 & 5.10 & 0.00 \\
\hline 06 & 6.60 & 0.00 \\
\hline 07 & 4.05 & 0.00 \\
\hline 08 & 19.23 & 19.00 \\
\hline 09 & 5.12 & 0.00 \\
\hline IO & 16.26 & 16.00 \\
\hline II & 5.09 & 5.00 \\
\hline I2 & $\mathrm{I} 4.22$ & I5.00 \\
\hline I3 & 6.09 & 6.04 \\
\hline I4 & 10.05 & 10.05 \\
\hline I5 & 2.00 & $4.0 \mathrm{I}$ \\
\hline I6 & 2O.II & $25 \cdot 33$ \\
\hline I7 & 15.05 & I5.I5 \\
\hline I8 & 20.00 & 21.40 \\
\hline I9 & 8.00 & 8.II \\
\hline 20 & 0.00 & $\mathrm{I} 4.25$ \\
\hline $2 \mathrm{I}$ & 0.00 & 9.12 \\
\hline 22 & 0.00 & 20.56 \\
\hline 23 & 0.00 & I5.37 \\
\hline
\end{tabular}

Fonte: Reynolds e Gutman (I988:23).

\section{d) Determinação das orientações de percepção dominantes}

A última fase do laddering consiste na análise das cadeias meios-fim originadas na etapa anterior e tem por objetivo verificar quais delas são dominantes, ou seja, quais delas mais contribuem para o resultado do mapa. Nessa etapa, as cadeias são analisadas desde a base (atributos), até o topo (valores pessoais), contando-se o número total de todas as relações diretas e indiretas existentes em cada possível seqüência $\mathrm{A}-\mathrm{C}-\mathrm{V}$. 
As cadeias com maiores somas de relações são consideradas as mais importantes. Entretanto, Leão e Mello (200I) alertam que é prudente que a análise seja feita de forma relativa e não absoluta. Na opinião dos autores não se deve levar apenas em consideração o número de relações, pois as cadeias com mais elementos tenderão a apresentar maior número de relações, o que não significa que são as mais importantes. Para eles (2001:13)

as cadeias com maior quantidade de elementos somarão, potencialmente, maior soma de relações se comparadas às cadeias com menor quantidade de elementos, o que não significa, necessariamente, serem mais importantes.

Um exemplo de como são determinadas as orientações de percepções dominantes é expressa na Tabela 3, que contém as interações entre todos os elementos na cadeia que se estende do elemento I (carbonação) até o elemento 23 (autoestima). A cadeia "carbonação (I)-auto-estima (23)" apresenta 64 relações diretas e 63 relações indiretas. Assim como foi feito na cadeia "carbonação (I)-autoestima (23)", deve-se fazer a contagem das relações em todas as demais cadeias, desde o nível de atributos até os valores pessoais. Finalmente, deve-se comparar o número total de relações apresentado por cada cadeia, verificando aquelas que mais se sobressaem e, conseqüentemente, que determinam as orientações de percepções dominantes.

\section{TABELA 3}

RELAÇÕES DA CADEIA “CARBONAÇÃO (I)-AUTO-ESTIMA (23)” PARA DETERMINAÇÃO DAS ORIENTAÇÕES DE PERCEPÇÕES DOMINANTES (REPRESENTATIVA)

\begin{tabular}{cccccccc}
\hline 0 & I & I0 & I2 & I6 & I8 & 23 & 0 \\
\hline I & 0.00 & I0.00 & 4.06 & 0.14 & 0.04 & 0.04 & I4.26 \\
\hline I0 & 0.00 & 0.00 & I0.00 & 5.10 & 0.06 & 0.02 & I5.18 \\
\hline I2 & 0.00 & 0.00 & 0.00 & I4.00 & 0.08 & 0.04 & I4.I2 \\
\hline I6 & 0.00 & 0.00 & 0.00 & 0.00 & I1.00 & I.05 & I2.05 \\
\hline I8 & 0.00 & 0.00 & 0.00 & 0.00 & 0.00 & 9.00 & 9.00 \\
\hline 23 & 0.00 & 0.00 & 0.00 & 0.00 & 0.00 & 0.00 & 0.00 \\
\hline 0 & 0.00 & 0.00 & 0.00 & 0.00 & 0.00 & 0.00 & 64.63 \\
\hline
\end{tabular}

Fonte: Reynolds e Gutman (I988:24). 


\section{ALGUMAS VARIANTES DO MÉTODO LADDERING}

O laddering envolve um processo trabalhoso que demanda dedicação, tempo e habilidade do pesquisador. Em função desses desafios, surgiram algumas iniciativas com o propósito de facilitar a implementação do método, o que culminou com variações do laddering. As propostas de variações do laddering referem-se: (i) à fase de coleta de dados, cuja proposta alternativa chama-se hard laddering, e (ii) à fase de análise e interpretação dos dados, que propõe a utilização de um programa computacional denominado LADDERMAP.

\section{a) Hard laddering}

O laddering tradicional é também chamado soft laddering e requer habilidade do pesquisador para ser implementado. O hard laddering é uma variação do método com maior estruturação na coleta de dados, que demanda menos habilidade do pesquisador durante a entrevista. Enquanto o soft laddering prioriza a livre expressão do respondente, tendo em vista apenas a pergunta básica "Por que isso é importante?", o hard laddering usa técnicas mais estruturadas de coleta de dados. Tem-se, como definição, que hard laddering se refere a técnicas de coleta de dados em que o respondente é forçado a produzir ladders um a um e a atribuir respostas de forma que a seqüência delas reflita aumentos nos níveis de abstração (GRUNERT; GRUNERT, I995). Grunert e Grunert (I995) explicam que, no geral, as técnicas de coleta de dados que não envolvem entrevistas pessoais se referem ao hard laddering. Para eles, questionários autoadministrados (como a versão papel e lápis do laddering) e mecanismos de coleta de dados computadorizados são exemplos dessa variação do método.

Gengler e Reynolds (I995) citam exemplos de propostas que visam facilitar a coleta de dados, utilizando o hard laddering. Entre elas, comentam sobre a tentativa de Gengler (I990), que usou um programa de computador para avaliar a força das associações entre conceitos que foram, a princípio, derivados de um focus group. Os autores também se referem à experiência de Valette-Florence e Rapacchi (I990), que usaram um cartão de escolha a partir de conceitos que foram relatados por um grupo.

Com base nessas experiências, Botschen, Thelen e Pieters (I999) afirmam que a vantagem do hard laddering é a eficiência na coleta de dados. Entretanto, eles advertem que pouco se conhece sobre a validade e a confiabilidade desse procedimento e de sua compatibilidade com os resultados obtidos por meio da entrevista laddering tradicional (soft laddering). Os autores recomendam o desenvolvimento de mais evidências que comparem as duas versões do método. Gengler e Reynolds (I995) acreditam que o hard laddering interfere em um dos 
pressupostos mais importantes do método, que é o de possibilitar a interface com as respostas criadas espontaneamente pelo entrevistado.

Em relação à comparação das abordagens, Grunert e Grunert (I995) asseveram que a abordagem soft é potencialmente melhor quando os tipos de problemas, no lado do respondente, são causados por estruturas cognitivas muito fracas ou muito elaboradas. Com efeito, eles acreditam que, quando o nível de conhecimento do respondente sobre um produto é muito baixo ou muito alto, o entrevistador deve preferir a soft laddering, por ter mais possibilidade de conduzir a entrevista de forma adequada. Além disso, a abordagem soft produz dados mais redundantes, o que facilita a reconstrução do significado na fase de análise de conteúdo e codificação. Por outro lado, quando a área pesquisada é bem conhecida e verifica-se que não há problemas na reconstrução dos significados, a hard laddering tem a vantagem de minimizar a influência do pesquisador.

\section{b) LADDERMAP}

O LADDERMAP é um software comerciável e constitui uma variação do método laddering tradicional por trazer inovações e algumas modificações no tratamento de dados; foi criado por Gengler e Reynolds (I995) para atuar como ferramenta de suporte à análise do laddering. Com o software, o julgamento do analista continua a ser muito importante, havendo apenas uma facilitação do trabalho operacional da análise (GENGLER; REYNOLDS, I995).

Lastovicka (I995) explica que os estágios da análise computacional do LADDERMAP compreendem quatro etapas, sendo elas: (i) entrada de dados e análise de conteúdo; (ii) geração da matriz de implicação; (iii) geração do mapa hierárquico de valor (HVM); e, por fim, (iv) arquivos para uso avançado.

Os criadores do software, Gengler e Reynolds (1995) esclarecem que o objetivo do primeiro estágio é especificar os elementos de uma cadeia meios-fim. Primeiramente, o analista deve separar as respostas de cada entrevistado em orações ou palavras-chave, para então entrar com esses dados brutos no programa. Ao entrar com os dados, o analista deve classificá-los em atributos, conseqüências ou valores pessoais. Essa classificação fundamenta a base teórica da análise e ajuda o analista a discernir o que são e não são palavras-chave relevantes. Logo, é definido um dicionário de códigos de conteúdo para realizar as classificações.

O programa auxilia a codificação dos elementos, sendo necessária a assinatura de cada oração dentro dos códigos. O software permite que o analista verifique o código atribuído a cada elemento e possibilita, inclusive, a conversão de orações com o mesmo sentido em sinônimos. O LADDERMAP agrupa os elementos de acordo com a dimensão a que eles pertencem. A tabulação da matriz de implicação envolve a definição de conexões entre os códigos de conteúdo.

No laddering tradicional, Reynolds e Gutman (I988) recomendam uma regra de decisão para determinar quais associações devem ser ilustradas no 
mapa hierárquico de valor (HVM). Essa decisão é a comparação de cada associação com um ponto de corte - se a associação entre duas dimensões aparecer em número maior ou igual ao ponto de corte, ela entra no mapa, caso contrário, não. O software implementa essa regra de decisão em seu algoritmo de construção HVM, que automaticamente realiza a construção de uma matriz de implicação agregada e faculta ao analista determinar um ponto de corte para selecionar as relações mais importantes. O LADDERMAP também permite que sejam geradas matrizes de implicações para segmentos diferentes, fazendo associações com códigos que se referem a aspectos demográficos dos entrevistados.

O desenho do mapa hierárquico de valor (HVM) é realizado a partir das definições estabelecidas no estágio anterior. Nessa etapa, Gengler e Reynolds (I995) comentam sobre um requerimento que é imposto ao analista: o HVM final deve representar um número significativo de associações derivadas dos dados brutos da entrevista laddering; a recomendação é que em torno de $70 \%$ das relações sejam apresentadas no mapa. $\mathrm{O}$ analista pode priorizar a exatidão dos dados, colocando o maior número de relações possíveis no mapa, pode priorizar a estética do mapa, deixando-o em um formato de fácil interpretação, ou pode decidir por um equilíbrio entre esses extremos.

Cabe destacar, por fim, que o LADDERMAP permite que os dados sejam analisados de diferentes formas, além das originalmente sugeridas por Reynolds e Gutman (1988). O software favorece associações com outros tipos de análise, como análise de correspondência (VALETTE-FLORENCE; RAPACCHI, I99I) e dendograma de conglomerado hierárquico (BHALLA, I989) - sendo os arquivos para uso avançado o último recurso proporcionado pelo LADDERMAP.

Ao fazer uma avaliação do software, Lastovicka (I995) conclui que é uma ferramenta útil que economiza tempo na análise do laddering. Contudo, o pesquisador faz algumas ressalvas. Os textos e instruções do LADDERMAP não são amigáveis, portanto o software é recomendado somente para uso daqueles que já estão familiarizados com o conceito do laddering e cadeias meios-fim. Sobre essa questão, o autor cita exemplos em que a terminologia do texto é diferente da opção real disponível no menu principal.

Outros pontos a destacar são que o mouse não pode ser utilizado, e isso dificulta a edição do HVM, e que os relatórios são sempre designados pelos mesmos nomes de arquivos fixos, o que impede que cópias prévias sejam armazenadas, pois o conteúdo do novo relatório é feito em cima do antigo. Além disso, o pesquisador comenta que os gráficos LADDERMAP, na maioria das vezes, têm de ser redesenhados para que haja um mínimo de clareza na interpretação. E comenta que talvez a maior limitação do programa seja o fato de as cadeias A$\mathrm{C}-\mathrm{V}$ produzidas pelo software não serem, necessariamente, apoiadas por ladders obtidos na resposta de um entrevistado, pois eles podem mesclar-se com as res- 
postas de outras pessoas, e, para um bom resultado, deve-se garantir que se trata de um grupo homogêneo.

\section{LIMITAÇÕES DO MÉTODO LADDERING}

As principais limitações do laddering são relativas à dificuldade de implementá-lo em larga escala, à necessidade de que os respondentes sejam em número reduzido, aos altos custos, ao tempo e à habilidade do pesquisador requeridos nas fases de entrevista e análise, à sua validade preditiva e, por fim, a aspectos relativos a procedimentos.

Vriens e Hofstede (2000) reforçam a restrição do método quanto à sua implementação em larga escala, por meio de amostras representativas. Como o laddering requer entrevistadores com habilidades qualitativas, uma implementação em larga escala poderia rapidamente tornar-se muito cara e demorar muito para ser completada. Essas considerações acabam recaindo sobre a necessidade de que os respondentes sejam em número reduzido para a implementação da técnica.

Tais idéias vêm ao encontro do pensamento de Botschen, Thelen e Pieters (I999). Os autores (I999) lembram que o soft laddering requer entrevistadores bem treinados para realizar a coleta de dados e que a análise de conteúdo demandada por esse método consome muito tempo do pesquisador, o que pode resultar em altos custos e complexidade.

É importante levar em consideração que, por abordar aspectos pessoais em diferentes níveis de abstração, a entrevista laddering pode apresentar dificuldades ao entrevistado, o que mais uma vez demanda habilidade de um pesquisador treinado para superar os bloqueios dos respondentes. Sobre essa questão, são dignas de nota as palavras de Gengler e Reynolds (I995:I9):

os dois maiores obstáculos que existem na proliferação do laddering como uma ferramenta gerencial são, primeiro, a magnitude do trabalho que o analista deve desempenhar e, segundo, o excessivo custo do estudo.

Gengler e Reynolds (I995) reconhecem as dificuldades do método relacionadas a custos, à demanda por pesquisadores habilitados e ao tempo gasto com as entrevistas. Os autores, inclusive, comentam sobre as possibilidades de reduzir esses obstáculos por meio da adoção de variantes do método laddering na fase de coleta de dados. No entanto, advertem que tais variações podem incidir em outros problemas, que colocam em risco a eficácia dos resultados e concluem que esses meios mais rápidos, mais baratos ou que requerem menos habilidade do pesquisador não são compensatórios. 
Grunert e Grunert (I995) avaliam a validade do laddering, partindo do pressuposto de que o método tem um objetivo preditivo, que é o de prescrever o comportamento de clientes, a partir do reconhecimento de suas estruturas cognitivas. Segundo suas palavras (I995:210):

O principal critério para avaliar a utilidade dessa abordagem [laddering] é sua habilidade preditiva de estimar a estrutura cognitiva obtida, quando usada como um input na teoria que explica o comportamento pelo qual, em uma dada situação, a informação é originada da estrutura cognitiva e usada na tomada de decisão. [...] O mapa de valor hierárquico derivado do laddering é um mapa de estruturas cognitivas agregadas. [...] As cadeias de meios-fim e o laddering objetivam mensurar as estruturas cognitivas do consumo relevante de consumidores.

Ao analisar a validade preditiva do laddering, os autores encontram vieses decorrentes de possíveis interferências do entrevistador na coleta de dados brutos e na análise de conteúdo. Em relação aos dados brutos, Grunert e Grunert (1995) consideram que o método não traz implicações negativas. Ainda que o pensamento do entrevistador possa ter considerável influência no curso da entrevista, ele não vai sugerir atributos, conseqüências e valores pessoais ao respondente, deixando-o suficientemente livre para usar sua própria estrutura cognitiva e responder às questões. Porém, em relação a outros aspectos da coleta de dados, o parecer desses pesquisadores não é o mesmo. Eles (I995) acreditam que, se o respondente tem pouco conhecimento do produto, mas o entrevistado o estimula a ir mais longe nos níveis de abstração, ele pode construir novas associações somente para se adequar às demandas da pesquisa. De forma análoga, afirmam que se o respondente tem bom conhecimento do produto pode encontrar dificuldades de seguir o formato hierárquico do laddering.

Além disso, Grunert e Grunert (I995) acreditam que, na análise de conteúdo, a interferência do pesquisador também pode ser fonte de viés. Ao codificar os elementos, o pesquisador tenta reconstruir o significado que o respondente atribuiu aos conceitos durante a entrevista. A dificuldade dessa tarefa depende da redundância dos dados - quando muitos respondentes indicam seqüências A$\mathrm{C}-\mathrm{V}$ parecidas, o trabalho de reconstruir a cadeia é mais fácil. Nessa fase, o pesquisador deve agrupar esses significados em categorias, o que abre espaço para a influência de sua própria estrutura cognitiva em relação aos dados da investigação. Nesse sentido, Chi-Feng (2002) também faz críticas à análise de conteúdo. Ele assevera que, ao se definirem os elementos, as variáveis são selecionadas e agrupadas segundo um processo subjetivo, que pode levar à eliminação de variáveis relevantes e, conseqüentemente, a estratégias de produto inapropriadas. 
Outra limitação apontada por Chi-Feng (2002) é o processo de simplificação das variáveis nas categorias atributos, conseqüências e valores pessoais. Apesar de necessário, esse processo pode restringir o escopo e a profundidade das respostas, o que poderia não refletir realmente os verdadeiros pensamentos dos entrevistados. No processo de codificação, Grunert e Grunert (I995) recomendam mais transparência e uso de métodos de análise de conteúdo assistidos por computador.

$\mathrm{Na}$ construção do mapa hierárquico de valor (HVM), Chi-Feng (2002) assinala que pode haver problemas na pré-definição do ponto de corte, o que também é motivo de crítica de Grunert e Grunert (I995), pois não existe um critério estatístico para selecionar o ponto de corte ideal. Grunert e Grunert (I995) explicam que os ladders, obtidos a partir da entrevista com um respondente individual, apenas revelam aspectos da estrutura cognitiva desse indivíduo, não representando a estimativa da estrutura cognitiva em si, pois ela não é uma simples coleção de cadeias, é sim uma rede inter-relacionada de associações.

Por outro lado, esses autores consideram que, num grupo homogêneo de respondentes, o conjunto de ladders obtidos, quando tomados juntos e analisados por um algoritmo apropriado, produz uma estimativa da estrutura cognitiva desse grupo como um todo. Isso explica o sentido da agregação de elementos na matriz de implicação e no mapa hierárquico de valor (HVM). Contudo, os autores (I995:22I) acreditam que essa agregação, em algumas situações, pode trazer problemas, como comentado no exemplo seguinte:

Imagine que, de uma amostra de 30 respondentes, 25 apresentam o ladder "gostar - sentir-se bem", e 5 apresentam o ladder "gostar - melhor função - sentir-se bem”. No mapa agregado, o grupo todo de respondentes poderia então ser caracterizado por "gostar - melhor função - sentir-se bem", porque uma ligação direta entre "gostar" e "sentir-se bem" seria redundante. Esse mapa, portanto, comunicaria uma impressão errônea que, para esse grupo de respondentes, o "gostar" leva a conseqüência pessoal da "melhor função" [...].

Na verdade, com o exemplo, Grunert e Grunert (I995) chamam a atenção para o fato de que nem sempre essa "regra" de agregação de elementos é válida. No caso apresentado, o mapa seria ilustrado por uma relação que não representa a estrutura cognitiva da maioria das pessoas pertencentes ao grupo, mas que, por causa da regra, ela seria priorizada em detrimento da outra. Os autores lembram que a decisão do ponto de corte é fundamental para evitar erros como esses. Essa limitação é compensada pelo uso do LADDERMAP, que proporciona alternativas de ponto de corte. 


\section{CONSIDERAÇÕES FINAIS}

Guardadas as limitações, o laddering se mostra um método vantajoso para a compreensão de comportamentos, que pode auxiliar diversas áreas de estudo relacionadas à administração, mas principalmente a do comportamento do consumidor em marketing. Há que se ter em mente, contudo, que essa não é a única técnica destinada a tal finalidade. Gengler, Mulvey e Oglethorpe (I999: I87) comentam que, apesar de a abordagem laddering ser indicada para descobrir insights em relação a comportamentos, existem outros métodos adequados para avaliar significados, tais como Metaphor elicitation (ZALTMAN, 1997), storytelling (THOMPSON, I997) ou ethnography (ARNOULD; WALLENDORF, I994).

O laddering pode ser considerado superior a outras técnicas quando se trata do estudo do valor segundo a teoria de cadeias meios-fim, por estruturar hierarquicamente as dimensões de valor e revelar seus níveis (atributos, conseqüências e valores pessoais) mais claramente. Ademais, constitui uma técnica completa que não se restringe à coleta de dados, pois também abrange sua análise e interpretação. Além disso, o fato de a análise seguir um mesmo formato (A-C-V) permite comparações mais diretas com outros segmentos em que o laddering também tenha sido aplicado, já que "a técnica facilita a comparação de resultados entre entrevistas, grupos-alvo e culturas" (DIBLEY; BAKER, 2001:82).

Mediante as informações apresentadas neste artigo, acredita-se que o uso do laddering deve levar em consideração alguns pontos em relação a suas etapas e variantes. Assim, recomenda-se:

- priorizar a qualificação do entrevistador e pesquisador, que devem dominar a teoria de cadeias meios-fim e possuir habilidades condizentes com a boa realização de entrevistas em profundidade e análise de dados qualitativos;

- verificar o nível de homogeneização do grupo de respondentes com o propósito de favorecer a redundância das respostas e facilitar a categorização dos elementos na etapa de análise de conteúdo;

- na etapa de coleta de dados, seguir as propostas sugeridas para a boa condução da entrevista, colocando em prática as técnicas evidenciadas por Reynolds e Gutman (I988) e as dicas de Wansink (2000);

- dedicar-se à fase de análise de conteúdo, a partir de exaustivas leituras, releituras e análise do material de transcrição das entrevistas;

- prezando pela fidedignidade dos resultados e seguindo a recomendação de Lastovicka (I995), minimizar os riscos envolvidos na construção da matriz de implicação com a utilização do LADDERMAP, que realiza todas as operações dessa fase da análise e interpretação dos dados, poupando o pesquisador 
do trabalho envolvido na construção da matriz e reduzindo as possibilidades de erros;

- para reduzir as possibilidades de dúvidas em relação ao ponto de corte ideal, utilizar o LADDERMAP na construção do mapa hierárquico de valor (HVM), que permite uma série de opções para a definição do ponto de corte, dispensando a elaboração da tabela que resume as relações diretas e indiretas dos elementos (veja Tabela 2);

- conforme Grunert e Grunert (I995), dar preferência ao laddering tradicional (soft laddering) quando o nível de conhecimento do respondente sobre o objeto é muito baixo ou muito alto e preferir o hard laddering quando a área pesquisada é bem conhecida e verifica-se que não há problemas na reconstrução de significados das respostas.

Finalizando, reforça-se o emprego do método laddering tanto em pesquisas acadêmicas como nas de mercado, acreditando em sua utilidade para desenvolvimento de estudos em administração e marketing.

\section{REFERÊNCIAS}

AAKER, D.; KUMAR, V.; DAY, G. Marketing research. Pesquisa de marketing. São Paulo: Atlas, 2OOI.

ARNOULD, E.; WALLENDORF, M. Market-oriented ethnography: interpretation building and marketing strategy formulation. Journal of Marketing Research, Chicago, v. 33, p. 484-504, Nov. I994.

BANNISTER, D.; MAIR, J. M. M. The evaluation of personal constructs. London: Academy Press, I968.

BHALLA, G. Mixing statistics with intuition. Paper presented at the AMERICAN MARKETING ASSOCIATION'S $20^{\text {th }}$ ANNUAL ATTITUDE RESEARCH CONFERENCE, Orlando, Florida, I989. Disponível em: <http://www.ama.org>. Acesso em: Io out. 2002.

BOTSCHEN, G.; THELEN, E. M.; PIETERS, R. Using means-end structures for benefit segmentation an application to services. European Journal of Marketing, Bradford, v. 33, n. I-2, p. 38-58, I999.

CHI-FENG, L. Attribute-Consequence-Value Linkages: a new technique for understanding customer's product knowledge. Journal of Targeting, Measurement and Analysis for Marketing, London, v. Io, n. 4, p. 339-352, June 2002.

DIBLEY, A.; BAKER, S. Uncovering the links between brand choice and personal values among young british and spanish girls. Journal of Consumer Behaviour, London, v. I, n. I, p. 77-93, June $200 I$.

GENGLER, C. E. An Architectural perspective on advertising strategy. Unpublished Doctoral Dissertation, University of Texas-Dallas, I990.

GENGLER, C. E.; MULVEY, M.S.; OGLETHORPE, J.E. A Means-end analysis of mother's infant feeding choices. Journal of Public Policy a Marketing, Ann Arbor, v. I8, n. 2, p. I72-I88, Fall I999. 
GENGLER, C. LADDERMAP User's Manual. Camden, New Jersey, I995.

GENGLER, C. E.; REYNOLDS, T. Consumer understanding and advertising strategy: analysis and strategic translation of laddering data. Journal of Advertising Research, New York, v. 35, n. 4, p. I9-32, July-Aug. I995.

GRUNERT, K. G.; GRUNERT, S. C. Measuring subjective meaning structures by the laddering method: theoretical considerations and methodological problems. International Journal of Research in Marketing, Amsterdam, v. I2, n. 3, p. 209-225, Oct. I995.

GUTMAN, J. A Means-end chain model based on consumer categorization processes. Journal of Marketing, Chicago, v. 46, p. 60-72, Apr. I982.

HINKLE, D. The change of personal constructs from the viewpoint of theory of construct implications. Unpublished PhD Thesis, Ohio State University, I965.

IGNÁCIO, C. P. O comportamento do consumidor de produtos alimentícios: um estudo exploratório sobre a importância das marcas. 2003. Dissertação (Mestrado em Administração) - Departamento de Administração da Pontifícia Universidade Católica de São Paulo. São Paulo: PUC, 2003.

KELLY, G. A. The psychology of personal constructs. New York: Norton \& Co, I955.

LASTOVICKA, J. L. LADDERMAP: version 4.0 by Chuck Gengler. Journal of Marketing Research, Chicago, v. 32, n. 4, p. 494-497, Nov. I995.

LEÃO, A. L. M. de S.; MELLO, S. C. B. Conhecendo o valor do cliente virtual: uma análise utilizando a teoria de cadeias de meios-fim. In: ENCONTRO DA ASSOCIAÇÃO NACIONAL DOS PROGRAMAS DE PÓS-GRADUAÇÃO EM ADMINISTRAÇÃO, 26, 2002, Salvador. Anais... Salvador: ANPAD, 2002.

; _. Mensurando customer value através do método laddering: uma proposta de aplicação da técnica para o ambiente virtual. In: ENCONTRO DA ASSOCIAÇÃO NACIONAL DOS PROGRAMAS DE PÓS-GRADUAÇÃO EM ADMINISTRAÇÃO, 25, 200I, Campinas. Anais... Campinas: ANPAD, 200I.

REYNOLDS, T. J.; GUTMAN, J. Laddering theory, method, analysis, and interpretation. Journal of Advertising Research, New York, v. 28, p. II-3I, Feb.-Mar. I988.

REYNOLDS, T.; WHITLARK, D. Applying laddering data to communications strategy and advertising practice. Journal of Advertising Research, New York, v. 35, n. 4, p. 9-I6, July-Aug. I995.

THOMPSON, C. Interpreting consumers: a hermeneutical framework for deriving marketing insights from the texts of consumer's consumption stories. Journal of Marketing Research, Chicago, n. 34, p. 438-455, Nov. I997.

VALETTE-FLORENCE, P.; RAPACCHI, B. Improvements in means-end chain analysis: using graph theory and correpondence analysis. Journal of Advertising Research, New York, p. 30-45, Feb.Mar. I99I.

VELUDO-DE-OLIVEIRA, T. M. Valor em serviços educacionais: um estudo sobre o marketing educacional no contexto da teoria de cadeias meios-fim. 2003. Dissertação (Mestrado em Administração) - Departamento de Administração da Faculdade de Economia, Administração e Contabilidade da Universidade de São Paulo. São Paulo: Universidade de São Paulo, 2003.

VRIENS, M.; HOFSTEDE, F. T. Linking attributes, benefits and consumer values. Journal of Marketing Research, Chicago, v. I2, n. 3, p. 4-Iо, Fall 2000.

WANSINK, B. New techniques to generate key marketing insights. Journal of Marketing Research, Chicago, v. I2, n. 2, p. 28-36, Summer 2000. 
WOODRUFF, R. B.; GARDIAL, Sarah F. Know your customer: new approaches to understanding customer value and satisfaction. Malden: Blackwell, I996.

ZALTMAN, G. Rethinking market research: putting people back in. Journal of Marketing Research, Chicago, n. 34, p. 424-437, Nov. I997.

T R A MITAÇÃ O

Recebido em 15/05/2004

Aceito em 10/08/2004 
Copyright of Revista de Administração Mackenzie is the property of Universidade Presbiteriana Mackenzie, RAM-Revista de Administracao Mackenzie and its content may not be copied or emailed to multiple sites or posted to a listserv without the copyright holder's express written permission. However, users may print, download, or email articles for individual use. 